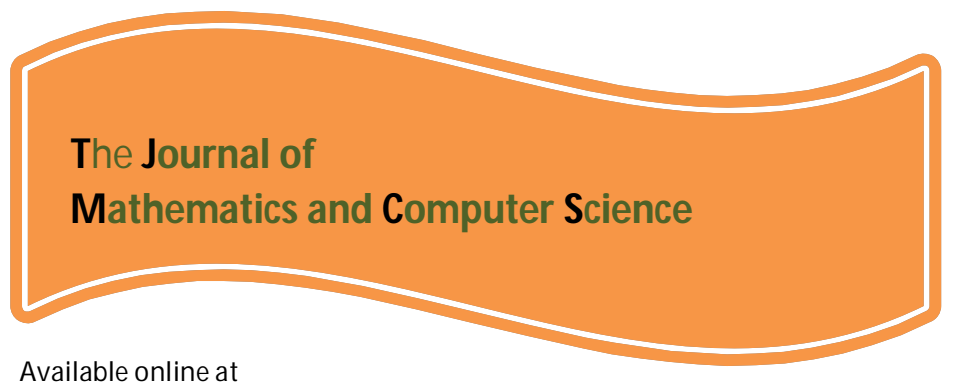

http://www.TJMCS.com

The Journal of Mathematics and Computer Science Vol. 4 No.3 (2012) 310 - 316

\title{
Symbolic computation and new soliton-like solutions to the
}

\author{
(2+1)-dimensional Toda lattice \\ Lan-Lan Huang, Kai-Teng Wu and Guo-Cheng Wu* \\ College of Mathematics and Information Science, Neijiang Normal University, \\ Sichuan 641112, P.R. China
}

Received: February 2012, Revised: May 2012

Online Publication: July 2012

\begin{abstract}
In this paper, with the aid of symbolic computation, an algebraic algorithm is proposed to construct soliton-like solutions to $(2+1)$-dimensional differentialdifference equations. The famous $(2+1)$-dimensional Toda equation is explicitly solved and some new classes of soliton-like solutions are obtained.
\end{abstract}

Keyword: symbolic computation; soliton-like solutions; non-travelling solution.

\section{Introduction}

Differential-difference equations (DDEs) play a crucial role in modeling of many physical phenomena such as particle vibrations in lattices, currents in electrical networks, pulses in biological chains, etc. Unlike difference equations which are fully discretized, DDEs are semi-discretized with some (or all) of their special variables discretized while time is usually kept continuous. Some aspects of DDEs,

*Corresponding author, Tel Numbers: 086-0832-2342745;

E-mail addresses: wuguocheng2002@yahoo.com.cn (G.C. Wu) 
such as integrability criteria, the computation of densities and symmetries, conservation laws and transformation theory, etc., have been studied extensively [1-6].

Not long ago, Baldwin et al [7]. presented an algorithm to find exact solutions of DDE in terms of tanh function and found kink-type solutions in many spatially discrete nonlinear models such as Hybrid lattice [7],Volterra lattice [8], discrete mKdV lattice [7, 9], Ablowitz-Ladik lattice [9], Toda lattice [10]. Then some extended algebraic methods [11 - 14] dedicate to seeking exact traveling solutions of DDE(s).

Recently, Zhu [15] extend the Exp-function method [16] and derived exact general solutions to the $(2+1)$ dimensional Toda lattice. The Exp method is proved to be nice and simple in seeking travelling solutions of DDES. But up to now, there are no algebraic methods to find non- traveling solutions.

In this paper, we propose an algorithm to solve $(2+1)$-dimensional DDEs and find exact non-traveling wave solutions. Our method is powerful when seeking for soliton-like solutions of NDDEs.

\section{Preliminaries}

Suppose the DDE we discuss here is given in the following nonlinear polynomial form

$$
\begin{aligned}
& H\left(u_{n+p_{1}}(t, x), \ldots, u_{n+p_{s}}(t, x), \frac{\partial u_{n+p_{1}}(t, x)}{\partial t}, \ldots, \frac{\partial u_{n+p_{1}}(t, x)}{\partial x}, \ldots, \frac{\partial u_{n+p_{s}}(t, x)}{\partial x}, \ldots,\right. \\
& \left.\frac{\partial u_{n+p_{1}}^{(m+x)}}{\partial t^{m} x^{r}}, \frac{\partial u_{n+p_{2}(t, x)}^{(m+r)}}{\partial t^{m} x^{r}}, \ldots, \frac{\partial u_{n+p_{s}}^{(m+x)}}{\partial t^{m} x^{r}}\right)=0 .
\end{aligned}
$$

where $u_{n}(t)=u(n, t)$ is a dependent variable; $t$ is a continuous variable; $n, m$, $P_{i} \in Z$.

To compute the non-travelling wave solutions to Eq. (1), we assume

$$
u_{n}(t, x)=u\left(\xi_{n}\right)
$$

where

$$
\xi_{n}=d n+r(t, x) .
$$

Step 1. Suppose that solutions to Eq. (1) satisfy the following form:

$$
\begin{aligned}
& u_{n+p_{i}}=\sum_{i=-N}^{N} a_{i}(t, x) \cosh ^{i}\left(\omega_{n+p_{i}}\right)+\sum_{i=1}^{N} b_{i}(t, x) \cosh h^{i-1}\left(\omega_{n+p_{i}}\right) \sinh \left(\omega_{n+p_{i}}\right) \\
& +\sum_{i=-N}^{-1} c_{i}(t, x) \cosh ^{i}\left(\omega_{n+p_{i}}\right) \sinh \left(\omega_{n+p_{i}}\right)
\end{aligned}
$$


and

$$
\frac{d \omega_{n}}{d \xi_{n}}=\sinh \left(\omega_{n}\right)
$$

where $a_{0}(t, x), \quad a_{ \pm 1}(t, x), \quad a_{ \pm N}(t, x), \quad b_{1}(t, x), \quad b_{N}(t, x), \quad c_{-1}(t, x)$ and $c_{-N}(t, x)$ are unknown functions to be determined later. $N$ can be determined by balancing the highest degree linear term and the nonlinear term of $u_{n}(t)$.

Meanwhile, if $\frac{d \omega}{d \xi}=\sinh (\omega)$, by using separation of variables method, we can derive

$$
\sinh (\omega)=-\operatorname{csch}(\xi) \text { and } \cosh (\omega)=-\operatorname{coth}(\xi) .
$$

When $p_{i}=0$, we can obtain

$$
\begin{aligned}
& u_{n}=\sum_{i=-N}^{N} a_{i}(t, x)\left(-\operatorname{coth}\left(\xi_{n}\right)\right)^{i}-\sum_{i=1}^{N} b_{i}(t, x)\left(-\operatorname{coth}\left(\xi_{n}\right)\right)^{i-1} \operatorname{csch}\left(\xi_{n}\right) \\
& -\sum_{i=-N}^{-1} c_{i}(t, x)\left(-\operatorname{coth}\left(\xi_{n}\right)\right)^{i} \operatorname{csch}\left(\xi_{n}\right)
\end{aligned}
$$

Step 2.: Simple computation leads to the following identity:

$$
\xi_{n+p_{i}}=\left(n+p_{i}\right) d+r(t, x)=\xi_{n}+d p_{i} .
$$

For $\sinh \left(\omega_{n}\right)=-\operatorname{csch}\left(\xi_{n}\right)$ and $\cosh \left(\omega_{n}\right)=-\operatorname{coth}\left(\xi_{n}\right)$, we can have

$$
\sinh \left(\omega_{n+p_{i}}\right)=-\operatorname{csch}\left(\xi_{n+p_{i}}\right)=\frac{\sinh \left(\omega_{n}\right)}{\cosh \left(d p_{i}\right)-\cosh \left(\omega_{n}\right) \sinh \left(d p_{i}\right)}
$$

and

$$
\cosh \left(\omega_{n+p_{i}}\right)=-\operatorname{coth}\left(\xi_{n+p_{i}}\right)=\frac{\cosh \left(\omega_{n}\right) \cosh \left(d p_{i}\right)-\sinh \left(d p_{i}\right)}{\cosh \left(d p_{i}\right)-\cosh \left(\omega_{n}\right) \sinh \left(d p_{i}\right)}
$$

Substituting (3) into Eq. (1), clearing the denominator, we obtain a finite series of $\sinh ^{k}\left(\omega_{n}\right) \cosh ^{i}\left(\omega_{n}\right)(i=0,1, \ldots, m, k=0,1)$. Setting the coefficients of $\sinh ^{k}\left(\omega_{n}\right) \cosh ^{i}\left(\omega_{n}\right)$ to zero, we get a set of difference differential equations with respect to the unknown $a_{i}, b_{i}$ and $c_{i}$

Step 3. Solve the nonlinear over-determined differential-difference equations. Explicit soliton-like solutions to Eq. (1) can be derived. 


\section{Non-traveling solutions of (2+1)-Dimensional Toda Lattice}

The $(2+1)$-dimensional Toda equation is

$$
\frac{\partial^{2} u_{n}(t)}{\partial t \partial x}=\left(\frac{\partial u_{n}(t)}{\partial t}+1\right)\left(u_{n-1}+u_{n+1}-2 u_{n}\right) .
$$

We assume soliton-like solutions to Eq. (8) in the form

$$
\begin{aligned}
& u_{n}=a_{0}(t, x)+\frac{a_{-1}(t, x)}{\cosh \left(\omega\left(\xi_{n}\right)\right)}+a_{1}(t, x) \cosh \left(\omega\left(\xi_{n}\right)\right)+b_{1}(t, x) \sinh \left(\omega\left(\xi_{n}\right)\right) \\
& +c_{-1}(t, x) \frac{\sinh \left(\omega\left(\xi_{n}\right)\right.}{\cosh \left(\omega\left(\xi_{n}\right)\right)}
\end{aligned}
$$

Eq. (9) can be rewritten as the following expression by means of Eq. (5),

$$
\begin{aligned}
& u_{n}=a_{0}(t, x)-a_{-1}(t, x) \tanh \left(\xi_{n}\right)-a_{1}(t, x) \operatorname{coth}\left(\xi_{n}\right)-b_{1}(t, x) \operatorname{csch}\left(\xi_{n}\right) \\
& +c_{-1}(t, x) \operatorname{sech}\left(\xi_{n}\right)
\end{aligned}
$$

Substituting Eq. (9) and Eq. (4) into Eq. (8), clearing the denominator and setting the coefficients of all powers like $\sinh ^{k}\left(\omega_{n}\right) \cosh ^{i}\left(\omega_{n}\right)$ $(i=0,1, \ldots, m, k=0,1$.) $\quad$ to zero, yields some over-determined difference differential equations with unknown functions with respect to $a_{-1}(t, x), a_{0}(t, x), a_{1}(t, x), b_{1}(t, x), c_{-1}(t, x)$ and $\xi_{n}(t, x)$.

We omit the equations for simplicity and solve the over-determined differential equations by the symbolic tool Maple. We can have the following fourteen classes of explicit non-traveling as

Familiy 1

$$
\begin{aligned}
& u_{n}=-t+F_{4}(x)-F_{2}(x) \tanh \left(\xi_{n}\right)-F_{3}(x) \operatorname{coth}\left(\xi_{n}\right)+F_{1}(x) \operatorname{sech}\left(\xi_{n}\right), \\
& \xi_{n}(t, x)=-2 \int F_{3}(x) d x+C_{1} ;
\end{aligned}
$$

Family 2. $u_{n}=-t+F_{2}(x)-F_{1}(x) \tanh \left(\xi_{n}\right), \xi_{n}(t, x)=F_{3}(x)$;

Family 3. $u_{n}=-t+F_{3}(x)-F_{2}(x) \tanh \left(\xi_{n}\right)+F_{1}(x) \operatorname{sech}\left(\xi_{n}\right), \xi_{n}(t, x)=F_{4}(x)$;

Family 4.

$u_{n}=-t+F_{5}(x)-F_{3}(x) \tanh \left(\xi_{n}\right)-F_{4}(x) \operatorname{coth}\left(\xi_{n}\right)-F_{2}(x) \operatorname{csch}\left(\xi_{n}\right)+F_{1}(x) \operatorname{sech}\left(\xi_{n}\right)$, $\xi_{n}(t, x)=-2 \int F_{4}(x) d x+C$;

Family 5 . 
$u_{n}=-t+F_{5}(x)-F_{3}(x) \tanh \left(\xi_{n}\right)-F_{4}(x) \operatorname{coth}\left(\xi_{n}\right)-F_{2}(x) \operatorname{csch}\left(\xi_{n}\right)+F_{1}(x) \operatorname{sech}\left(\xi_{n}\right)$, $\xi_{n}(t, x)=2 \int\left(F_{2}(x)-F_{4}(x)\right) d x+C ;$

Family 6.

$u_{n}=-t+F_{4}(x)-F_{2}(x) \tanh \left(\xi_{n}\right)-F_{3}(x) \operatorname{coth}\left(\xi_{n}\right)+F_{1}(x) \operatorname{sech}\left(\xi_{n}\right)$,

$\xi_{n}(t, x)=F_{5}(x)$

Family 7.

$u_{n}=-t+F_{5}(x)-F_{3}(x) \tanh \left(\xi_{n}\right)-F_{4}(x) \operatorname{coth}\left(\xi_{n}\right)-F_{2}(x) \operatorname{csch}\left(\xi_{n}\right)+F_{1}(x) \operatorname{sech}\left(\xi_{n}\right)$,

$\xi_{n}(t, x)=F_{6}(x)$;

Family 8.

$u_{n}=F_{2}(t)+F_{1}(x)-C_{1} \operatorname{coth}\left(\xi_{n}\right), \xi_{n}=d n-C_{1} x-\frac{(\cosh (2 d)-1)\left(t+F_{2}(t)\right)}{C_{1}}+C_{2} ;$

Family 9.

$u_{n}=F_{2}(t)+F_{1}(x)-C_{1} \tanh \left(\xi_{n}\right), \xi_{n}=d n-C_{1} x-\frac{(\cosh (2 d)-1)\left(t+F_{2}(t)\right)}{C_{1}}+C_{2} ;$

Family 10.

$u_{n}=F_{2}(t)+F_{1}(x)-C_{1} \tanh \left(\xi_{n}\right)-C_{1} \operatorname{coth}\left(\xi_{n}\right), \xi_{n}=d n-C_{1} x-\frac{(\cosh (4 d)-1)\left(t+F_{2}(t)\right)}{8 C_{1}}+C_{2} ;$

Family 11.

$u_{n}=F_{4}(t)+F_{3}(x)+i C_{1} \tanh \left(\xi_{n}\right)+C_{1} \operatorname{sech}\left(\xi_{n}\right), \xi_{n}=d n+2 i C_{1} x-\frac{(\cosh (d)-1)\left(t+F_{4}(t)\right) i}{C_{1}}+C_{2} ;$

Family 12.

$u_{n}=F_{4}(t)+F_{3}(x)-i C_{1} \tanh \left(\xi_{n}\right)+C_{1} \operatorname{sech}\left(\xi_{n}\right), \xi_{n}=d n-2 i C_{1} x+\frac{(\cosh (d)-1)\left(t+F_{4}(t)\right) i}{C_{1}}+C_{2} ;$

Family 13.

$u_{n}=F_{4}(t)+F_{3}(x)-C_{1} \operatorname{coth}\left(\xi_{n}\right)-C_{1} \operatorname{csch}\left(\xi_{n}\right), \xi_{n}=d n-2 C_{1} x-\frac{(\cosh (d)-1)\left(t+F_{4}(t)\right)}{C_{1}}+C_{2} ;$

Family 14.

$u_{n}=F_{2}(t)+F_{1}(x)+C_{1} \operatorname{coth}\left(\xi_{n}\right)-C_{1} \operatorname{csch}\left(\xi_{n}\right), \xi_{n}=d n+2 C_{1} x+\frac{(\cosh (d)-1)\left(t+F_{4}(t)\right) i}{C_{1}}+C_{2}$.

$F_{i}(t), F_{i}(x)$ and $C_{i}$ are arbitrary in all the above fourteen families. 
Consider the solution Family 10. Setting $C_{1}=C_{2}=1, d=\operatorname{arctanh}\left(\frac{\sqrt{2}}{2}\right), n=100$, $F_{2}=\operatorname{sech}(t), F_{1}(x)=\tanh (x)$, Family 10 reads

$$
u_{100}=\operatorname{sech}(t)+\tanh (x)-\tanh \left(\xi_{n}\right)-\operatorname{coth}\left(\xi_{n}\right)
$$

where $\xi_{n}=100 \operatorname{arctanh}\left(\frac{\sqrt{2}}{2}\right)-x-\frac{\left(\cosh \left(4 \operatorname{arctanh}\left(\frac{\sqrt{2}}{2}\right)\right)-1\right)(t+\operatorname{sech}(t))}{8}$.

The exact solution Family 10 is illustrated in the Fig 1.

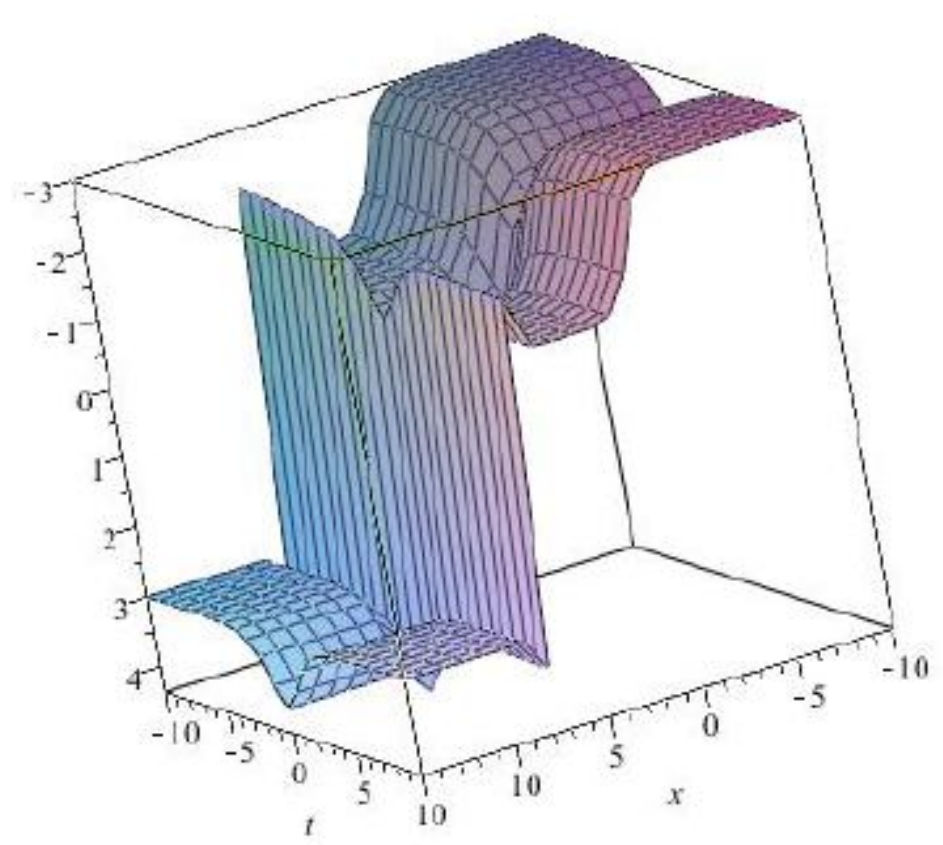

Fig 1. The shape of Family 10 with $F_{1}(x)=\tanh (x), F_{2}(t)=\operatorname{sech}(t), d=\operatorname{arctanh}\left(\frac{\sqrt{2}}{2}\right)$

Families of solutions 8-14 aren't linear functions depended on $t$ and they are more general than constant ones. As we known, Family 2, Family 8, Family 9 and Family10 can be found in Ref. [17]. Other ten Families presented in this paper have not been found in previous literature. This paper also provides some exact solutions of the Toda lattice for various nonlinear approximate techniques [18-22].

\section{Conclusions}

With symbolic computation, when applying our proposed method to Eq. (1), a 
rich variety soliton-like solutions given in forms of sech, tanh, coth, csch. Our method can also be used to construct non-traveling solutions of other higher dimensional DDE(s).

\section{References}

[1]D. Levi, O. Ragnisco, Lett. Nuovo. Cimento. 1978; 22: 691-696.

[2]D. Levi, R. I. Yamilov, J. Math. Phys. 1997; 38: 6648-6674.

[3] R. I. Yamilov, J Phys A: Math Gen 1994; 27: 6839-6851.

[4]Y. B. Suris J Phys A: Math Gen 1997; 30: 1745-1761.

[5]M. Wadati, Watanabe M. Prog Theor Phys 1977; 57: 808-811.

[6]M.J. Wadati, Phys Soc Jpn 1976; 40: 1517-1518.

[7]D. Baldwin, Goktasu, Hereman W. Comput Phys Commun 2004; 162: 203-217.

[8] V. E. Adler, Svinolupov SI, Yamilov RI. Phys LettA1999; 254: 24-36.

[9] M. J. Ablowtiz, J. F. Ladik JF. Stud Appl Math 1997; 57: 1-12.

[10] M. Toda.J Phys Soc Jpn, 1967; 22: 431-436;

M. Toda. Sup Prog Theor Phys, 1976; 59: 1-35;

M. Toda. Theory of nonlinear lattices. Berlin Heidelberg: Springer-Verlag; 1988.

[11]F. D. Xie, J. Q. Wang, Chaos, Soliton. Fractal. 2006; 27: 1067-1071.

[12]Z. Wang, H. Q. Zhang, Chaos, Soliton. Fractal. 2007; 33: 642-654.

[13] S. Zhang, Q. A. Zhong, D. Liu, Q. Gao, Commun. Frac. Calc. 2011; 1: 48-51.

[14] Z. Wang, W.X. Ma, Math. Meth. Appl. Sci. 2010; 33: 1463-1472.

[15] S. D. Zhu, Int.J. Nonlin. Sci. Num. Sim. 2007; 8: 465-468.

[16] J. H. He, X.H. Wu, Chaos, Soliton. Fractal. 2006; 30: 700-706.

[17] G. C. Wu, T. C. Xia, Chaos, Soliton. Fractal. 2009; 39: 2245-2248.

[18] F. Mehrdoust, H. Aminikhah, M. Ghamgosar, J. Math. Comput. Sci. 2011; 3: 376-381.

[19] M. Alipour, D. Rostamy, J. Math. Comput. Sci. 2011; 3: 403-412.

[20] J. H. He, Int. J. Nonlinear Mech. 1999; 34: 699-708.

[21] J. H. He, Appl. Math. Comput. 2002; 135: 73-79.

[22] H. Jafari, S. A. Yousefi, M. A. Firoozjaee, S. Momani, Comput. Math. Appl. 2011; 62: 1038-1045. 